\title{
TRANSPORTE PÚBLICO E SUSTENTABILIDADE: UMA ANÁLISE DAS PUBLICAÇÕES À LUZ DA BIBLIOMETRIA
}

\section{PUBLIC TRANSPORT AND SUSTAINABILITY: AN ANALYSIS OF PUBLICATIONS IN THE LIGHT OF BIBLIOMETRY}

\author{
Tálita Floriano Santos* E-mail: talitafloriano@pet.coppe.br \\ Marcelino Aurélio Vieira Silva*E-mail: marcelino@pet.coppe.ufri.br \\ * Universidade Federal do Rio de Janeiro (UFRJ), Rio de Janeiro, RJ
}

\begin{abstract}
Resumo: A relação entre o transporte público e a sustentabilidade tem se destacado nas pesquisas científicas. Assim, o objetivo desse artigo é utilizar as abordagens da bibliometria e redes sociais para analisar as publicações relacionadas a esses temas. Para bibliometria foram utilizados critérios de produtividade dos periódicos, autores e citações de publicações oriundas das bases Science Direct, Scopus e Web of Science. Foram selecionados 48 artigos se destacando os que tratavam da caracterização do padrão de viagens e escolha modal, técnicas para melhorias do transporte público, sustentabilidade ambiental e social, sendo a maioria $(81 \%)$ de aplicação prática. A análise de rede social, feita pelo CiteSpace, abordou 245 referências, utilizando as métricas de modularidade, silhueta e centralidade, para autores citados, países e palavras chave. Os países com maiores centralidades são: Estados Unidos, China e Austrália, mas a Inglaterra e Estados Unidos contêm as áreas de pesquisa mais ativas. Para as palavras chave, as maiores centralidades foram "sustainable development", "built environment" e "travel behavior". Os autores com maiores centralidades foram o Cervero R. e Banister D., apresentando convergência com o que foi encontrado na bibliometria. Por fim, o estudo mostrou que a análise bibliométrica e de rede social, são complementares e convergentes, ambas indicando tendências de literatura a sustentabilidade ambiental e social.
\end{abstract}

Palavras-Chave: Transporte Público. Sustentabilidade. Bibliometria. Indicadores Espaciais. Transporte Sustentável.

\begin{abstract}
The relationship between public transport and sustainability has been highlighted in scientific research. Thus, the purpose of this article is to use the approaches of bibliometrics and social networks to analyze the publications related to these subjects. For bibliometrics, we used productivity criteria of journals, authors and citations of publications from the bases Science Direct, Scopus and Web of Science. A total of 48 articles were selected, highlighting those that dealt with the characterization of travel patterns and modal choice, techniques for public transport improvements, environmental and social sustainability, with the majority $(81 \%)$ being of practical application. The analysis of social network, made by CiteSpace, approached 245 references, using the metrics of modularity, silhouette and centrality, for cited authors, countries and keywords. The countries with the largest centralities are the United States, China and Australia, but England and the United States contain the most active research areas. For key words, the major centralities were "sustainable development", "built environment" and "travel behavior". The authors with the greatest centralities were Cervero R. and Banister D., showing convergence with what was found in bibliometrics. Finally, the study showed that bibliometric and social network analysis are complementary and convergent, both indicating trends in the literature on environmental and social sustainability.
\end{abstract}

Keywords: Public Transport. Sustainability. Bibliometrics. Space Indicators. Sustainable Transport. 


\section{INTRODUÇÃO}

É reconhecido que os cidadãos precisam planejar o sustento do planeta e que os sistemas de transporte exercem um papel importante nessa questão, seja pela questão da sustentabilidade, seja por eles promoverem o acesso às oportunidades econômicas e sociais necessárias para uma vida significativa (Richardson, 2005). Assim, o desenvolvimento de um sistema de transporte público adequado é fundamental para o desenvolvimento sustentável (Murray et al., 1998).

Dessa forma, o desenvolvimento sustentável e transporte estão conectados: cidades em torno do mundo vivenciam congestionamentos dado o aumento do número de automóveis, que lideram as emissões de gases prejudiciais ao meio ambiente, além dos custos sociais relacionados (Miller et al., 2016).

Sob essa perspectiva, em 2015, as Nações Unidas desenvolveram 17 metas que objetivam alcançar uma sociedade mais sustentável e um mundo pacífico no ano de 2030 e a expansão do transporte urbano é um dos maiores desafios (Alpkokin et al., 2016; De Gruyter et al., 2017).

Diante dessa complexidade, as redes de transporte representam um paradoxo: elas promovem o desenvolvimento urbano, mas por outro lado apresentam uma série de desafios (econômicos, sociais e impactos ambientais) (Miller et al., 2016), sendo uma relação de trade-off que precisa ser entendida para posteriormente, ser analisada e gerenciada da melhor forma possível. Essa relevância é ratificada, pelo número de publicações crescentes da relação entre transporte público e sustentabilidade.

No entanto, devido ao grande número de publicações esse artigo tem por objetivo identificar como as principais pesquisas sobre Transporte Público estão relacionadas com os aspectos da sustentabilidade por meio de uma revisão bibliométrica realizada em três bases de dados (Science Direct, Scopus e ISI Web of Knowledge). Além disso, faz também uma análise de rede social, através das redes de co-citação dos países, palavras chave e autores através do software CiteSpace, com base no Web of Knowlegde. 


\section{TRANSPORTE PÚBLICO E SUSTENTABILIDADE}

Transporte sustentável inclui todos os aspectos que estão relacionados ao transporte, como: proteção da fauna, redução dos níveis de ruído, promoção do crescimento econômico, redução dos níveis de congestionamento, minimização dos acidentes e mortes, aumento da contribuição do turismo no PIB, promoção de ruas e bairros habitáveis e minimização da criminalidade relacionada com o transporte (Holden et al., 2013). Devido a esse amplo espectro, o transporte sustentável apresenta desafios.

Alcançar a sustentabilidade econômica, social e ambiental do transporte urbano é um dos grandes desafios contemporâneos (Alpkokin et al., 2016), podendo se dar especialmente pelo uso de transporte não motorizado (caminhadas e bicicletas) ou motorizados através do transporte público.

Assim, as políticas de sustentabilidade dos transportes urbanos visam incentivar uma maior utilização dos transportes públicos e uma melhoria da prestação de serviços (Alpkokin et al., 2016) e por conta da necessidade de se promover o transporte público, o governo precisa desenvolver programas, políticas e estratégias para auxiliar a operação do TP, aumentar sua competitividade e atrair mais passageiros (Buehler, 2009). Com essa necessidade de promoção ao TP, por conta das questões voltadas a sustentabilidade, é necessário discutir a conscientização dos usuários para permitir uma mudança de comportamento ligada a dependência do automóvel.

Uma suposição implícita subjacente às estratégias governamentais para sistema de transporte urbano sustentável é que todos os usuários de automóveis serão encorajados ou persuadidos a usar mais transporte "verde": transporte público, caminhadas e ciclismo (Nakanishi e Black, 2015), sendo que quase todos esses contextos estão voltados paara reduzir as emissões de dióxido de carbono $\left(\mathrm{CO}_{2}\right.$.) (Hickman et al., 2013).

Essas questões estão relacionadas a um melhor uso do solo e a uma melhor infraestrutura no sistema de transporte, sendo um verdadeiro desafio implementá-las (Hickman et al., 2013). 


\section{BIBLIOMETRIA}

Muito se tem discutido sobre a árdua e necessária tarefa de mensurar, caracterizar e avaliar a ciência, ou seja, verificar o resultado da atividade intelectual de pesquisadores e estudiosos, que têm seu produto apresentado de diversas maneiras (Araújo e Alvarenga, 2011). Diante disso, a bibliometria apresenta-se como uma técnica quantitativa que pode ser utilizada.

A bibliometria foi inicialmente voltada para a medida de livros e aos poucos foi se voltando para o estudo de outros formatos de produção bibliográfica, como artigos de periódicos e outros tipos de documentos, para depois ocupar-se, também, da produtividade de autores e do estudo de citações (Araújo, 2006), sendo indicada para análise de portfólios muito extensos, onde a seleção de maneira qualitativa torna-se demasiadamente demorada (Arantes e Santos, 2016).

Uma das formas de realizar a análise bibliométrica dos artigos é pela a lei de produtividade de autores de Lotka e a lei de dispersão de periódicos de Bradford.

A Lei de Lotka, formulada em 1926, foi construída a partir de um estudo sobre a produtividade de cientistas, em que ele constatou que uma larga proporção da literatura científica é produzida por um pequeno número de autores, e um grande número de pequenos produtores se iguala, em produção, ao reduzido número de grandes produtores (Araújo, 2006).

A Lei de dispersão de Bradford constata que ordenando uma grande coleção de periódicos em ordem de produtividade decrescente relevante a um dado assunto, três zonas aparecem, cada uma contendo $1 / 3$ do total de artigos relevantes (a primeira zona contém um pequeno número de periódicos altamente produtivos, a segunda contém um número maior de periódicos menos produtivos, e a terceira inclui mais periódicos ainda, mas cada um com menos produtividade).

Assim, a bibliometria é uma ferramenta que pode ser utilizada quando há necessidade de se avaliar uma grande quantidade de artigos, de forma que a avaliação apenas qualitativa se torna inviável. 


\section{ANÁLISE DE REDE SOCIAL}

A partir do início da década de 1970, houve um aumento no número de trabalhos técnicos e aplicações de especialistas relacionados com as análises redes sociais (Social Network Analysis) e assim, essas técnicas foram gradualmente incorporadas massivamente a análise de dados e uma esfera mais ampla de aplicações pelo meio científico (Scott, 2013).

As redes sociais são uma analogia importante pera se compreender fenômenos sociais complexos, uma vez que permitem aos pesquisadores trabalharem com um grande volume de variáveis e com inúmeras relações entre elas (Neder e Marques, 2015). Para isso, existem métricas importantes que auxiliam na análise dessas redes: modularidade (modularity), silhueta (silhouette) e centralidade (centrality ou betweenness).

A modularidade mede o grau em que uma rede pode ser dividida em blocos ou módulos independentes, associada as propriedades estruturais da rede, com pontuação variando de 0 a 1 , sendo que uma baixa modularidade sugere uma rede que não pode ser reduzida a clusters com limites claros, enquanto uma alta modularidade pode indicar uma rede bem estruturada (Chen et al., 2010). Uma baixa modularidade pode dificultar as análises das redes pois tornaria difícil encontrar padrões para o estudo, ou ainda, os textos não fazem parte da mesma área científica (Neder e Marques, 2015).

A métrica silhueta é útil na estimativa da incerteza envolvida na identificação da natureza de um aglomerado de vértices. O valor da silhueta de um clustervaria de -1 a 1 e indica a incerteza que precisa ser levada em conta na interpretação da natureza do cluster científica (Neder e Marques, 2015). A rotulagem de cluster ou a agregação de outras tarefas se tornará mais simples para clusters com o valor silhueta na faixa de 0,7 $\sim$,9 ou superior (Chen et al., 2010).

Por fim, a métrica de centralidade pode ser definida para cada nó da rede, sendo medida a partir do grau em que o nó está no meio do percurso que o liga aos outros vértices desta rede. Valores altos para essa métrica podem identificar publicações científicas potencialmente revolucionárias. (Chen et al., 2010).

As pontuações de centralidade de intermediação são normalizadas para a unidade de intervalo de $[0,1]$. Um nó de alta centralidade de intermediação é 
geralmente um que liga dois ou mais grandes grupos de nós com o próprio nó no meio, por isso o termo intermediação (Chen, 2014).

\section{MÉTODO DE PESQUISA}

\subsection{Coleta de Dados}

Para a coleta dos dados, foram selecionadas as bases de dados ISI Web of Science, Science Direct e Scopus, realizando-se em duas etapas. A primeira etapa foram inseridas as palavras chaves "Sustainability" e "Public Transport", e a segunda com "Sustanability" e "Public Transportation" para aparecem como tópico ou resumo.

A busca no ISI Web of Science resultou em 166 artigos, na base Scopus 656 e na Web of Science, 302 artigos, totalizando 1.124. Com o objetivo de analisar apenas artigos que tenham sido revisados por pares, excluiu-se os artigos não oriundos de periódicos, sendo eliminados 301 artigos, restando assim 823.

Como as bases possuem periódicos em comum, são encontrados títulos repetidos que devem ser eliminados antes de iniciar a análise bibliométrica. Esta verificação foi feita com o suporte do software Mendeley e Endnote. Assim, foram eliminados 322 artigos, restando 501 oriundos de periódicos e sem repetições.

Posteriormente, procedeu-se com a leitura dos títulos para verificar se os artigos estavam alinhados com o tema da pesquisa. Dos 501 artigos, foram eliminados 63, restando 438 artigos de periódicos, sem repetições e com título alinhado ao tema da pesquisa. Ou seja, o portfólio para início da análise bibliométrica possui 438 artigos. Essas etapas são descritas na Figura 1. 
Figura 1- Etapas da Coleta de dados da Bibliometria

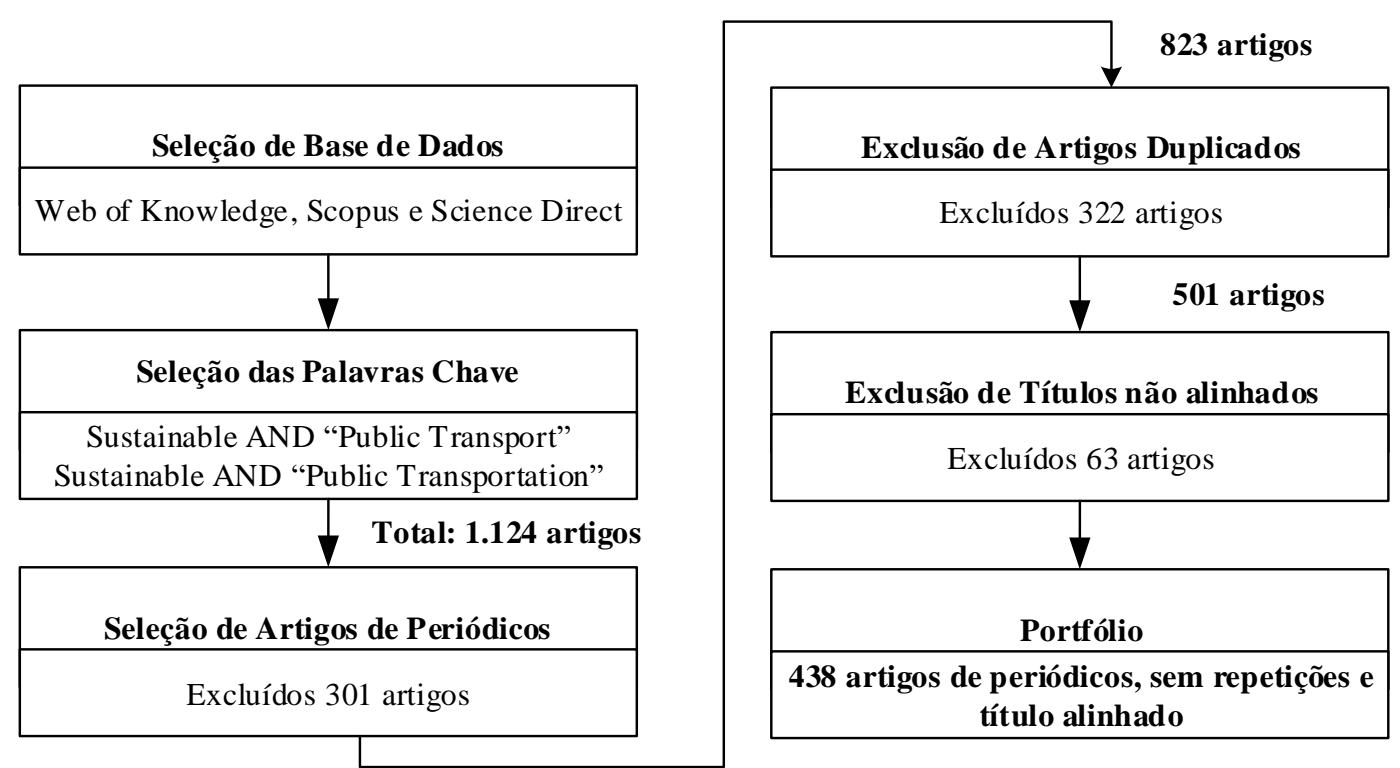

Fonte: Elaboração Própria

Assim, conforme mostra a Figura 1, as etapas para coleta de dados foram: seleção da base de dados, seleção das palavras chave, seleção de artigos oriundos de periódicos, exclusão de artigos duplicados e não alinhados, e com isso, tem-se o portfólio para análise bibliométrica.

\subsection{Análise de Dados}

A análise dos dados se procedeu de duas formas. A primeira através da bibliometria (descrita no tópico 4.2.1) e uma espacial (descrita no tópico 4.2.2).

\subsubsection{Bibliometria}

Para análise dos dados, pela bibliometria, o primeiro fator observado foi a produtividade dos periódicos. Estes são listados, relacionando a quantidade de artigos publicada em cada um e a representatividade de cada periódico em termos percentuais. Em seguida, avalia-se a produtividade dos autores, identificando (1) o número de trabalhos publicados por cada autor, (2) o percentual de autores com apenas uma publicação e (3) destacando aqueles com mais pesquisas publicadas.

Finalmente, verifica-se o número de citações total de cada artigo e a média de citações por ano, tendo como base o tempo de publicação. Para esta análise, foi 
utilizado o indicador do Google Acadêmico, uma vez que foram usadas três bases de dados e a contagem de citações das mesmas não pode ser comparada por serem realizadas de maneiras diferentes.

Assim, esses indicadores (produtividade dos periódicos, produtividade dos autores, número de citações total e número médio de citações por ano) são analisados conjuntamente, em que se estabelece um ponto de corte para cada indicador, realizando assim a análise bibliométrica das publicações.

\subsubsection{Análise de Rede Social}

A Social Network Analysis (SNA) feita com o software CiteSpace permite a importação dos dados da base Web of Science (WoS), com isso, obteve-se 246 artigos de periódicos para análise no período de 1995 a 2017.

Foram feitas análises baseadas em três nós: países, palavras chave e autores citados. Para esses nós foram gerados clusters, em que foram analisadas as métricas de modularidade, silhueta e centralidade.

Posteriormente, foram feitas análises dos clusters mais relevantes para cada um dos nós. Finalmente, foi verificado se existe explosão (burst) em algum dos nós obtidos pela base de dados do WoS.

\section{RESULTADOS E DISCUSSÕES}

Pela Figura 1, a relação entre sustentabilidade e transporte público começou a ser publicada no final da década de 1990, ganhando força após 2010, com crescimento observado em praticamente todos os anos. A Figura 1 mostra esse desenvolvimento, com a média móvel sendo mostrada pela linha pontilhada. 
Figura 2 - Quantidade de publicações por ano

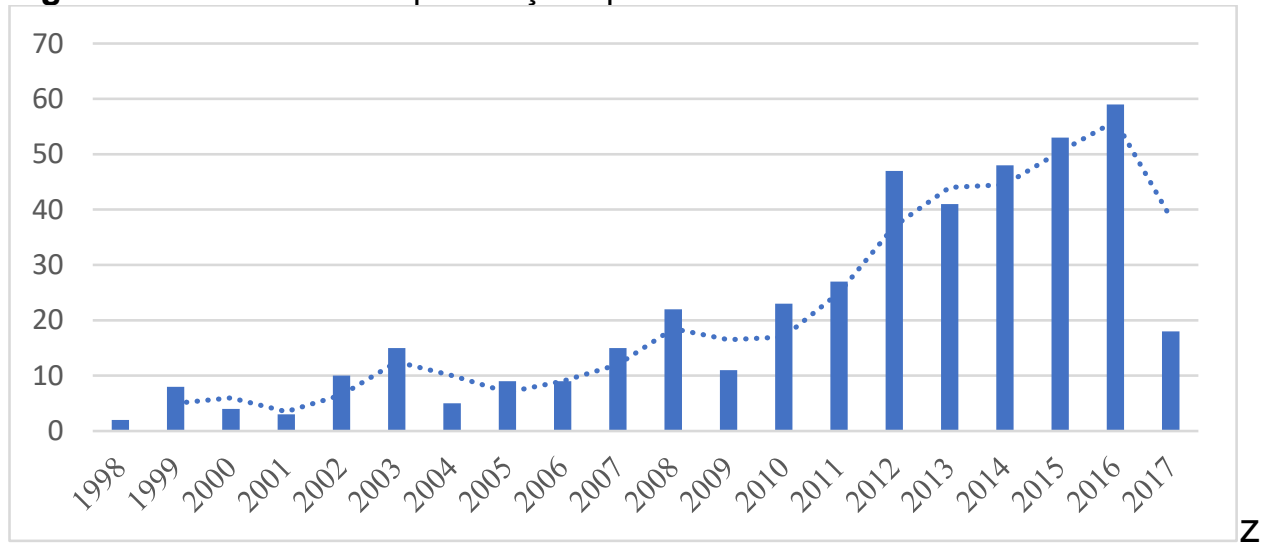

\subsection{Produtividade dos Periódicos}

Os 438 artigos do portfólio final foram encontrados em 206 periódicos. Destes, apenas 15 deles são responsáveis por 40,41\% das publicações, a saber: Transport Policy, Journal of Transport Geography, Research in Transportation Economics, Transportation Research Record, Transportation Research Part A: Policy and Practice, Transportation Research Part D: Transport and Environment, WIT Transactions on Ecology and the Environment, Transportation Research Procedia, International Journal of Sustainable Transportation Transportation, Sustainability (Switzerland), Journal of Cleaner Production, Transport Reviews, Habitat International e Public Transport International. A Tabela 1 apresenta um gráfico com todos os periódicos e a sua relação em percentual.

Tabela 1- Periódicos com maior representatividade no portfólio

\begin{tabular}{lccc}
\hline \multicolumn{1}{c}{ Periódico } & Qtd & $\%$ & \% \\
\hline Transport Policy & 26 & 5,94 & 5,94 \\
Journal of Transport Geography & 15 & 3,42 & 9,36 \\
Research in Transportation Economics & 15 & 3,42 & 12,79 \\
Transportation Research Record & 15 & 3,42 & 16,21 \\
Transportation Research Part A: Policy and Practice & 14 & 3,20 & 19,41 \\
Transportation Research Part D: Transport and Environment & 14 & 3,20 & 22,60 \\
WIT Transactions on Ecology and the Environment & 14 & 3,20 & 25,80 \\
Transportation Research Procedia & 11 & 2,51 & 28,31 \\
International Journal ofSustainable Transportation & 10 & 2,28 & 30,59 \\
Transportation & 9 & 2,05 & 32,65 \\
Sustainability (Switzerland) & 8 & 1,83 & 34,47 \\
Journal of Cleaner Production & 7 & 1,60 & 36,07 \\
Transport Reviews & 7 & 1,60 & 37,67 \\
Habitat International & 6 & 1,37 & 39,04 \\
Public Transport International & 6 & 1,37 & 40,41 \\
\hline
\end{tabular}

Fonte: Elaboração própria 
Pela Tabela 1, é possível visualizar que o periódico Transport Policy é o que tem um maior número de publicações com 26 artigos publicados. Além disto, dentro do portfólio, 3 periódicos apresentaram 5 publicações cada, 10 apresentaram 3, 29 apresentaram 2, e por fim, 146 periódicos apresentaram apenas 1 publicação, ratificando assim com a lei de Bradford de que um pequeno número de periódicos concentra a maior parte das pesquisas.

\subsection{Produtividade dos Autores}

A segunda parte da revisão bibliométrica consiste em analisar a produtividade dos autores. Foram identificados 997 autores, dos quais 916 possuem apenas uma publicação, representando aproximadamente 92\%. Um autor possui 7 publicações, 2 possuem 5 publicações, 4 autores possuem 4, 12 possuem 3 e 62 autores possuem 2 publicações.

Os autores que possuem maior número de publicações tendem a desenvolver um conhecimento mais aprofundado sobre o tema, e assim, espera-se importantes contribuições desses para o Transporte Público e a Sustentabilidade. A

Figura 3 mostra os autores do portfólio que tiveram pelo menos 3 publicações.

Figura 3 - Principais autores

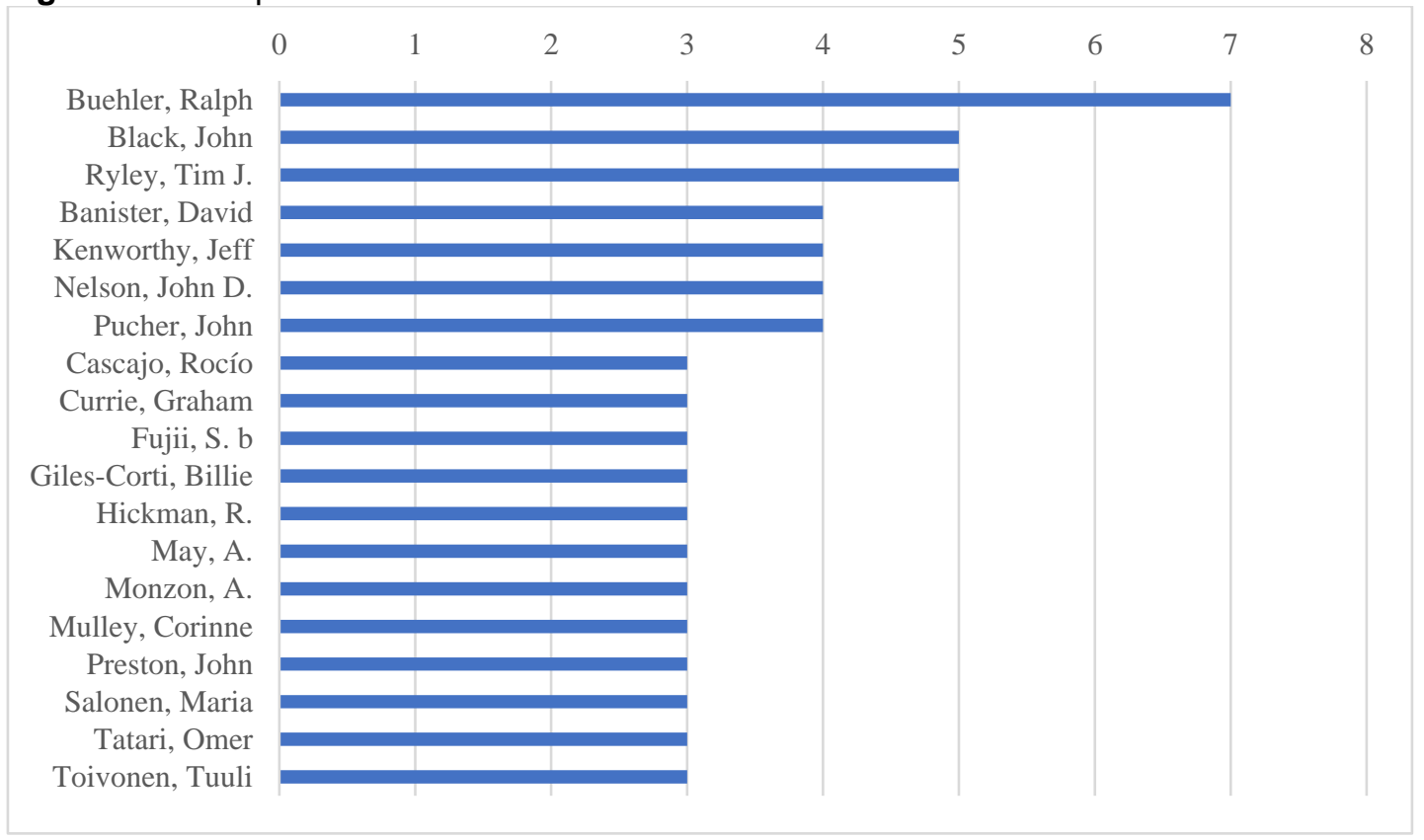

Fonte: Elaboração própria 
Pela Figura 3, pode-se visualizar que o autor com maior número de publicações é R. Buehler com 7 publicações, seguido por J. Black e T. J. Ryley, com 5 publicações cada. A

Tabela 2 mostra o número de contribuições (publicações) por autor e a quantidade de autores em cada uma destas.

\begin{tabular}{ccc}
\multicolumn{3}{c}{ Tabela 2- Número de contribuições por autor } \\
\hline № de contribuições por autor & № de autores & \% de autores \\
\hline 7 & 1 & 0,1 \\
5 & 2 & 0,2 \\
4 & 4 & 0,4 \\
3 & 12 & 1,2 \\
2 & 62 & 6,2 \\
1 & 916 & 91,9 \\
Total & 997 & 100
\end{tabular}

Fonte: Elaboração própria

\subsection{Produtividade das Citações}

Além da produtividade dos periódicos e dos autores, é importante analisar a produtividade das citações que pode ser feita de duas formas: citação total ou por ano. A primeira analisa o número de citações total de um artigo, analisado pelo Google Acadêmico. A segunda realiza a divisão entre o número total de citações e seu ano de publicação, por exemplo: se um artigo foi publicado em 2017 e ele apresenta 10 citações no total, então sua citação anual é 10. Porém, se um artigo é de 2016 e apresenta 10 citações no total, sua citação anual é 5. A Tabela 3 exibe os autores que alcançaram uma produtividade de ao menos 10 citações por ano. 
Tabela 3- Produtividade das citações

\begin{tabular}{|c|c|c|c|c|}
\hline \multirow{2}{*}{ Referência } & \multicolumn{2}{|c|}{ Citação } & \multicolumn{2}{|c|}{ Ranking baseado em } \\
\hline & Total & Por ano & Total & Por ano \\
\hline (Shaheen et al., 2010) & 416 & 52 & 2 & 1 \\
\hline (Camagni et al., 2002) & 572 & 35,8 & 1 & 2 \\
\hline (Santos et al., 2010) & 233 & 29,1 & 6 & 3 \\
\hline (Kenworthy, 2006) & 328 & 27,3 & 3 & 4 \\
\hline (Buehler e Pucher, 2012) & 158 & 26,3 & 12 & 5 \\
\hline (Chen et al., 2008) & 254 & 25,4 & 5 & 6 \\
\hline (Haapio, 2012) & 131 & 21,8 & 15 & 7 \\
\hline (Buehler, 2011) & 142 & 20,3 & 14 & 8 \\
\hline (Nair et al., 2013) & 120 & 20 & 18 & 9 \\
\hline (Carlsson-Kanyama et al., 2008) & 167 & 16,7 & 10 & 10 \\
\hline (Richardson, 2005) & 209 & 16,1 & 8 & 11 \\
\hline (Giles-Corti et al., 2016) & 32 & 16 & 77 & 12 \\
\hline (Buehler e Pucher, 2011a) & 108 & 15,4 & 21 & 13 \\
\hline (Kok e Jennen, 2012) & 88 & 14,67 & 26 & 14 \\
\hline (Salonen e Toivonen, 2013) & 71 & 14,20 & 33 & 15 \\
\hline (Murray et al., 1998) & 274 & 13,70 & 4 & 16 \\
\hline (Joss e Molella, 2013) & 67 & 13,40 & 35 & 17 \\
\hline (Viegas, 2001) & 222 & 13,06 & 7 & 18 \\
\hline (Black et al., 2002) & 207 & 12,94 & 9 & 19 \\
\hline (Hensher, 2008) & 129 & 12,9 & 16 & 20 \\
\hline (Buehler e Pucher, 2011b) & 86 & 12,3 & 27 & 21 \\
\hline (Meinhold et al., 2013) & 61 & 12,2 & 39 & 22 \\
\hline (Murphy e Usher, 2015) & 34 & 11,3 & 71 & 23 \\
\hline (Cherry e Cervero, 2007) & 124 & 11,3 & 17 & 24 \\
\hline (Brida et al., 2014) & 45 & 11,3 & 51 & 25 \\
\hline (Shiftan et al., 2003) & 166 & 11,1 & 11 & 26 \\
\hline (Caniels e Romijn, 2008) & 107 & 10,7 & 23 & 27 \\
\hline (Chester et al., 2013) & 52 & 10,4 & 46 & 28 \\
\hline (Curtis, 2008) & 101 & 10,1 & 24 & 29 \\
\hline (Zhou, 2012) & 60 & 10 & 40 & 30 \\
\hline (Miller et al., 2015) & 30 & 10 & 81 & 31 \\
\hline (Buehler et al., 2017) & 10 & 10 & 172 & 32 \\
\hline
\end{tabular}

Fonte: Elaboração própria

A Tabela 3 mostra que o autor R. Buehler além de ser o autor mais produtivo do portfólio (vide Figura 3), aparece 4 vezes nos artigos com maiores citações. Todos os artigos tiveram sua citação contabilizada, em que o somatório foi de 8.417 citações. 
Os 32 artigos listados na Tabela 3 apresentam um total de 4804 citações, representando mais de $57 \%$ de todas as citações.

Por fim, para se estabelecer quais artigos devem ser selecionados, foi estabelecido um ponto de corte para cada indicador (periódicos, autores e citações). Foi constatado que os periódicos mais produtivos são os que possuem ao menos 6 publicações, representando 40,41\% das publicações. Assim, o ponto de corte para os periódicos aqueles que possuem 6 publicações ou mais.

Para o segundo indicador (autores), foi estabelecido o seguinte ponto de corte: foram considerados aqueles com 3 ou mais publicações. Por fim, o terceiro indicador (citações), foram definidos aqueles com mais de 10 citações por ano. Com o intuito de não excluir artigos recentes (publicados em 2016 e 2017) foi estabelecido o ponto de corte de 5 citações por ano. Dessa forma, foram considerados os artigos que atenderam dois ou mais desses critérios e com isso, obteve-se 48 artigos selecionados.

\subsection{Análise das palavras chave}

Com auxílio do Endnote, foram identificadas 195 palavras chave nos artigos selecionados. No entanto, 5 deles não apresentaram palavras chave $(10,4 \%$ da amostra). As palavras chave com maior frequência foram: Sustainability (10), Public Transport (8), Land use (4), Public Transit (3), Transport (3), Sustainable Transportation (3), Germany (3), Efficiency (3) e Australia (3), que mostram convergência com os temas principais dos artigos.

A análise das palavras chave auxiliou a identificar os sub temas dos artigos, ou seja, a ideia central de cada. Os sub temas selecionados fazem referências às principais abordagens dos artigos: caracterização do padrão de viagens relacionado a escolha modal, abordagens/técnicas voltadas para melhoria do TP, Parceria Público-Privada para financiamento do TP, Política de Restrição para uso do automóvel, Dependência do automóvel, Utilização e/ou compartilhamento de bicicletas, tecnologia aplicada à melhoria do transporte, indicadores de sustentabilidade, sustentabilidade ambiental, sustentabilidade social, sustentabilidade financeira. 


\subsection{Caracterização da Amostra}

Devido a amplitude do tema, os artigos selecionados apresentam as abordagens mais diversas. No entanto, 39 artigos dos 48 apresentam aplicação prática, representando mais de $81 \%$ da amostra.

Um dos pontos de destaque é a utilização de técnicas voltadas para melhoria do transporte público. Diante dessa preocupação, é necessário abordar um sistema de transporte sustentável, com políticas voltadas ao TP e incentivos a modos não motorizados que são apresentados pelos mais diversos autores (Alpkokin, Kiremitci, et al., 2016; Buehler e Pucher, 2011a; Giles-Corti et al., 2016; Kenworthy, 2006; May et al., 2000, 2011; Shiftan et al., 2003), sendo assim importante destacar as publicações que tratam de modos de transporte motorizados mais sustentáveis como trens e ônibus.

Assim, o ônibus deve também ser levantado quanto a uma solução mais sustentável aos modos de transporte (Finn e Nelson, 2002), tendo a Alemanha consigo tornar isso sustentável financeiramente (Buehler e Pucher, 2011b), levantando questões acerca da sustentabilidade financeira em transporte público e também a necessidade de parcerias público-privadas para sua viabilidade (Black et al., 2002; May et al., 2000, 2011).

Quanto ao uso do transporte não motorizado, a bicicleta apresentou bastante relevância no portfólio. Seja pela realidade do compartilhamento de bicicletas (Nair et al., 2013; Shaheen et al., 2010), pelo uso de bicicletas elétricas (Cherry e Cervero, 2007) ou outras análises sobre ciclovias e a infraestrutura necessária (Buehler e Pucher, 2012; Murphy e Usher, 2015), mostrando que elas são fundamentais para promoção de um transporte mais sustentável.

Outro ponto interessante, é a preocupação com o comportamento, envelhecimento das pessoas e como isso pode ser afetado pelo sistema de transporte, através da mudança de hábitos durante vários estágios da vida (Abou-Zeid e Fujii, 2016; Currie e Delbosc, 2010; Nakanishi e Black, 2016), trazendo uma análise do padrão de viagens relacionado a escolha modal e a sustentabilidade social. 


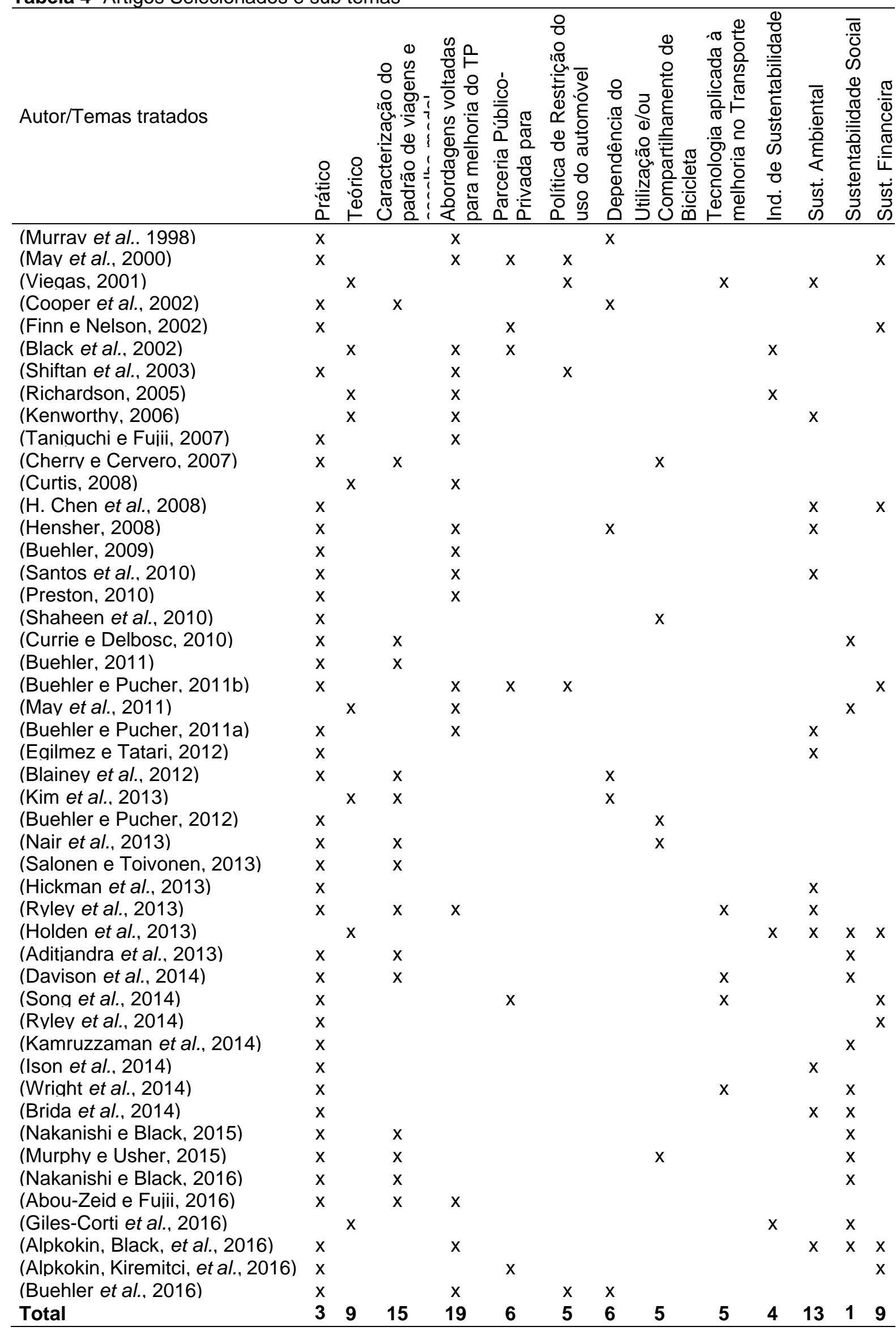

Revista Produção Online. Florianópolis, SC, v. 19, n. 1, p. 44-71, 2019. 
A sustentabilidade social também é discutida seja como um fator para promoção do turismo com o uso de teleféricos (Brida et al., 2014), ou pela sua eficiência com perspectivas de equidade (Viegas, 2001), inclusive com as motoristas mais idosos (Nakanishi e Black, 2015).

Com isso, além da necessidade de promover ações, também se faz necessário quantificá-las, que se dá por meio de indicadores de sustentabilidade (Black et al., 2002; Richardson, 2005). Assim, percebe-se a amplitude do tema, bem como a importância do transporte público para o desenvolvimento sustentável. Assim, uma síntese dos temas que foram abordados na amostra selecionada é mostrada na Tabela 4.

\subsection{Análise de Redes sociais}

Para análise de rede social, foi utilizado o software CiteSpace, utilizando a base de dados Web of Knowledge (WoS) - sendo essa a sua principal base de dados com as mesmas palavras chave da análise bibliométrica. Com isso, obteve-se 246 artigos de periódicos, no período de 1995 a 2017.

O software permite a escolha de vários tipos de nós (node types) para se fazer a análise, tais como: autor, país, palavra chave, referências citadas, etc. Para este trabalho, foram selecionados os seguintes node types: países, palavras chave e autores citados.

Foram utilizados três indicadores: clustering, burst e centrality. O clustering fornece uma maneira precisa para identificar grupos (clusters), usando a função de agrupamento do software que apresenta três métricas importantes que foram analisadas neste trabalho: a modularidade (modularity), a silhueta (silhouette) e a centralidade (centrality) do cluster.

\subsubsection{Análise por Países}

Uma das formas de se obter as informações é por meio de clusters que permite visualizar e identificar alguns tipos de grupos proeminentes. Para os países, foram identificados um total de 10 clusters, com 23 países. Cada um desses grupos corresponde a um fio condutor, um tópico ou uma linha de pesquisa. 
Foi mostrada uma modularidade de $\mathrm{Q}=0,38$, considerada razoavelmente baixa o que significa que a rede está razoavelmente dividida em clusters de baixo acoplamento, sendo um indicio que não existe necessariamente um país padrão para o estudo. A métrica silhueta de pontuação de 0,27 sugere que homogeneidade destes aglomerados, em média, não é muito elevada, apresentando um indicativo de que os clusters não serão facilmente rotulados. A Tabela 5 mostra a quantidade de publicações por países, sua respectiva contagem e centralidade.

Tabela 5- Quantidade de Publicações e centralidade por país

\begin{tabular}{clc}
\hline Contagem & \multicolumn{1}{c}{ País } & Centralidade \\
\hline 41 & Estados Unidos & 0,19 \\
30 & Inglaterra & 0,07 \\
22 & Austrália & 0,17 \\
20 & China & 0,19 \\
16 & Holanda & 0,03 \\
10 & Espanha & 0,01 \\
7 & Suécia & 0,00 \\
7 & Alemanha & 0,07 \\
6 & Canadá & 0,03 \\
6 & Itália & 0,00 \\
5 & Brasil & 0,03 \\
5 & Suíça & 0,13 \\
4 & Japão & 0,00 \\
4 & Índia & 0,00 \\
3 & Turquia & 0,00 \\
3 & Irã & 0,00 \\
2 & Portugal & 0,00 \\
2 & Finlândia & 0,00 \\
2 & Taiwan & 0,00 \\
2 & Malásia & 0,00 \\
2 & Chile & 0,00 \\
2 & Irlanda do Norte & 0,00 \\
2 & Escócia & 0,00 \\
\hline
\end{tabular}

Fonte: Elaboração Própria

Os países com as maiores centralidades são: Estados Unidos, China e Austrália, ou seja, são os principais países que se ligam a outros clusters. Outro indicador do CiteSpace identifica onde estão as áreas de pesquisa mais ativas, que se dá por meio da detecção de explosão (burst). Para a análise dos países, o CiteSpace detectou explosão na Inglaterra e Estados Unidos, sendo um no período 
de 1997 a 2007 e outro no período de 2003 a 2012, respectivamente, conforme mostrado na Figura 4.

Figura 4- Histórico de Explosão na Inglaterra e nos Estados Unidos

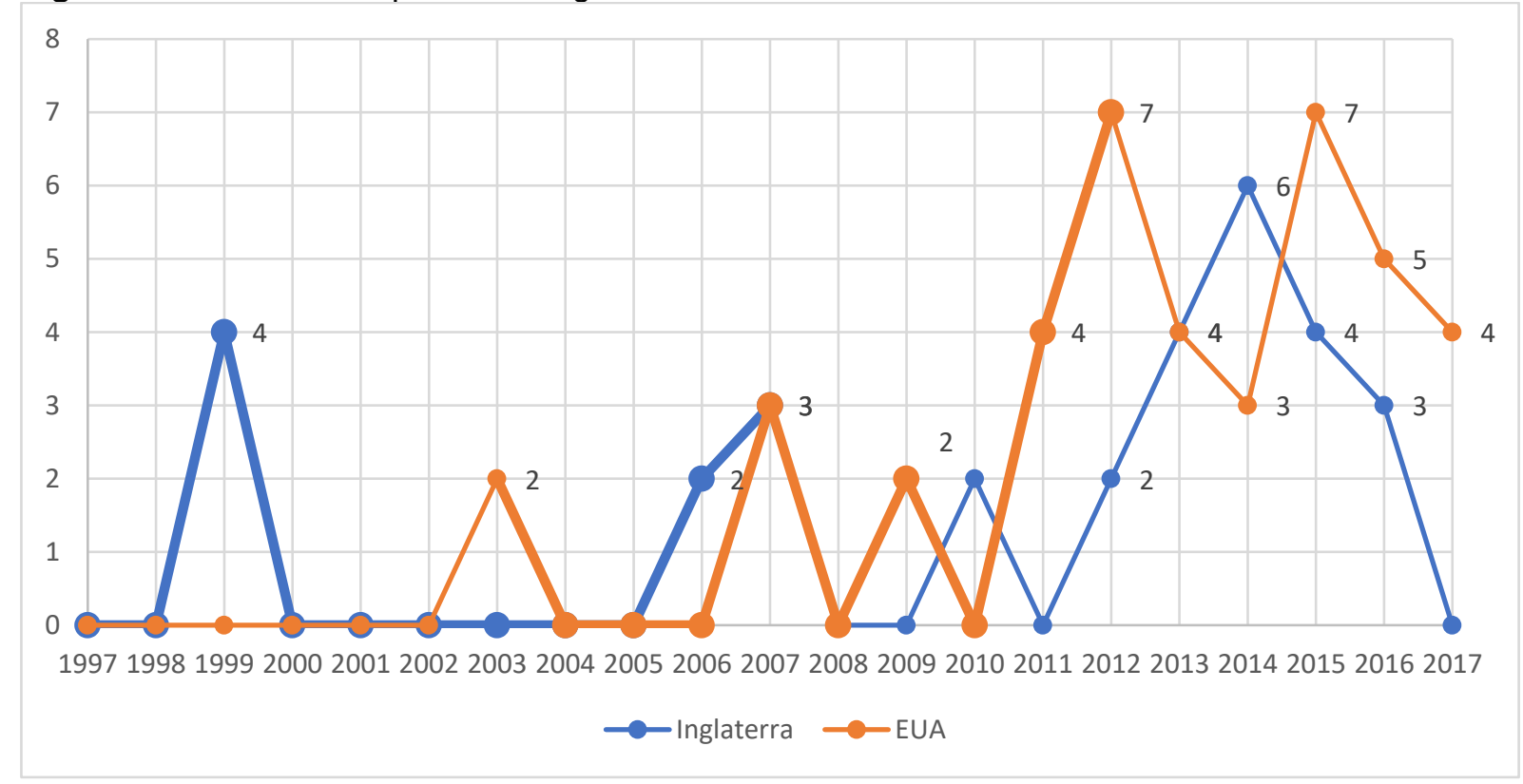

\subsubsection{Análise por Palavras Chave}

A segunda análise foi feita selecionando outro tipo de nó (node type): keywords. Para as palavras chave, foram identificados 10 clusters com 99 palavras chave. Foi mostrada uma modularidade de $Q=0,4533$, considerada razoavelmente baixa. $A$ métrica silhueta de pontuação de 0,4573 sugere que homogeneidade destes aglomerados não é muito elevada. A Tabela 6 mostra a quantidade de publicações das principais palavras chave detectadas. As maiores centralidades em ordem decrescente são: sustainable development $(0,18)$, built environment $(0,16)$, travel behavior $(0,16)$, public transport $(0,16)$, climate change $(0,15)$, policy $(0,14)$, mode choice $(0,13)$, urban form $(0,13)$ e walking $(0,12)$. As palavras chave apresentadas com maiores centralidades indicam tendências da literatura. 
Tabela 6- Quantidade de Publicações e Centralidade por Palavras Chave

\begin{tabular}{clc}
\hline Contagem & Palavra chave & Centralidade \\
\hline 75 & sustainability & 0,11 \\
48 & Public transport & 0,16 \\
21 & Transport & 0,04 \\
17 & System & 0,07 \\
16 & City & 0,11 \\
14 & Land use & 0,04 \\
13 & Transportation & 0,01 \\
12 & Impact & 0,08 \\
12 & Public transportation & 0,11 \\
11 & Policy & 0,14 \\
10 & Model & 0,04 \\
10 & Sustainable development & 0,18 \\
\hline
\end{tabular}

Fonte: Elaboração própria

Para caracterizar a natureza de um cluster identificado, após testes, foi escolhido para gerar clusters a partir das palavras chave. Os maiores clusters indicados por palavras-chave, em ordem decrescente são: "atitude", "neighborhood", "accessibility", "sustainable development", "bicycle", "cycling", "compact city" e "transport planning". Esses clusters indicam as principais áreas de pesquisa de acordo com as palavras chave da base WoS.

Outra função utilizada foi a detecção de explosão (burst), em que foi encontrada uma palavra chave de uma área de pesquisa mais ativa: sustainability, com início em 1997 e término em 2011, apresentando uma relação com outras palavras chave, como: energy, policy, impact, pattern, bicycle, public transport, germany system e technology. A Figura 5 mostra o histórico da explosão da palavra-chave sustentability.

Figura 5- Histórico da Explosão (Sustentability)

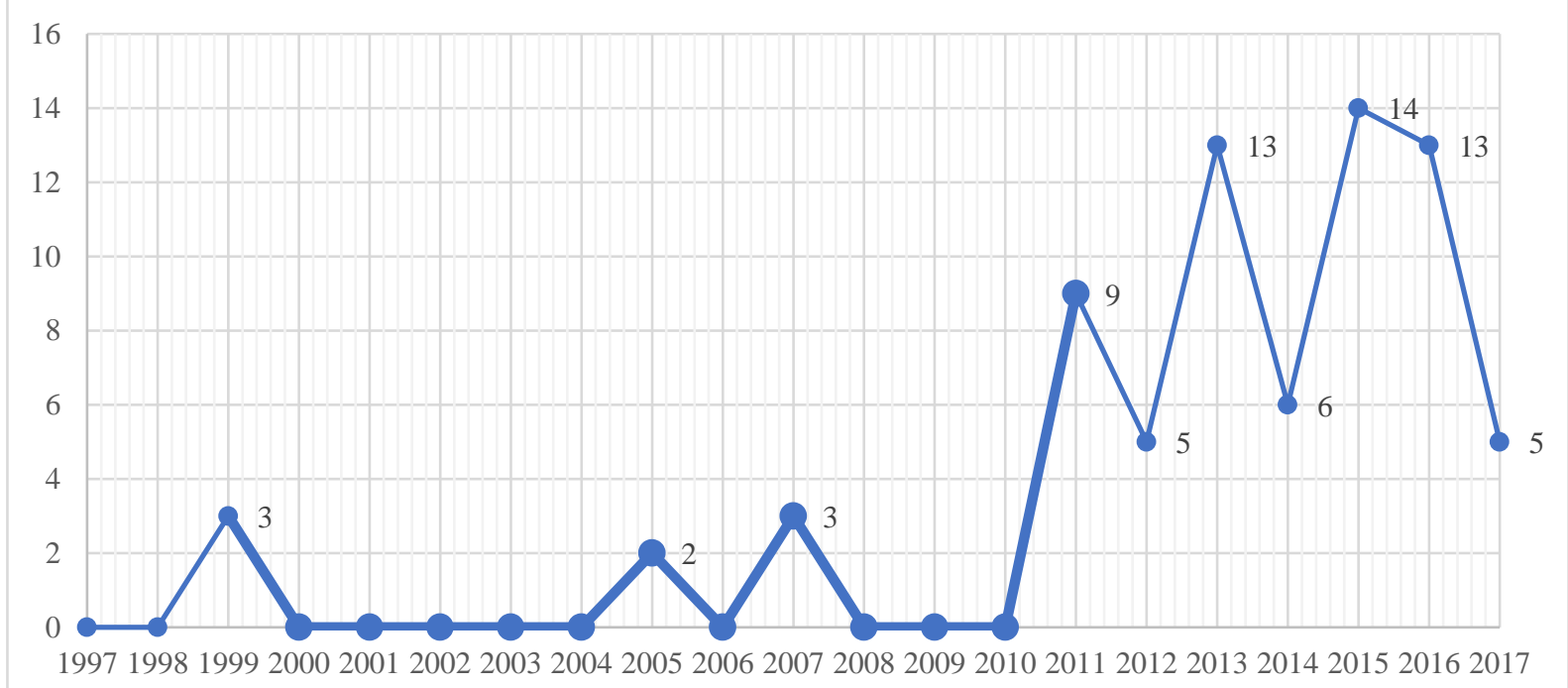

Fonte: Elaboração própria 
Pela Figura 6, nota-se que mesmo após o ano de 2011, ela ainda apareceu várias vezes, mas não é considerada um "boom", já que se manteve entre altos e baixos nos anos seguintes.

\subsubsection{Análise por autores citados}

Por fim, a última análise foi feita selecionando o nó de autores. Para esse item, foram identificados 6 clusters. Foi mostrada uma modularidade de $Q=0,67$, considerada relativamente alta, o que indica uma rede bem estruturada, enquanto a silhueta retornou o valor 0,24 . As maiores centralidades foram dos autores Cervero e Banister, apresentando convergência com o que foi encontrado pela bibliometria. A Tabela 7 apresenta os principais autores citados, sua contagem e centralidade.

\begin{tabular}{clc}
\multicolumn{3}{c}{ Tabela 7- Principais autores citados e Centralidade } \\
\hline Contagem & Palavra chave & Centralidade \\
\hline 41 & Cervero R & 0,39 \\
36 & Banister D & 0,23 \\
26 & Pucher J & 0,10 \\
21 & Litman T & 0,08 \\
20 & Newman P & 0,06 \\
18 & Buehler R & 0,10 \\
17 & Ewing R & 0,12 \\
12 & Schwanen T & 0,05 \\
11 & Hensher DA & 0,05 \\
\hline
\end{tabular}

Fonte: Elaboração própria

Por fim, foi utilizada a função de deteç̧ão de explosão. Os autores citados que se sobressaíram foram Newman P. e Pucher J, conforme mostra a Figura 7. 
Figura 6- Histórico de Explosão de Autores Citados

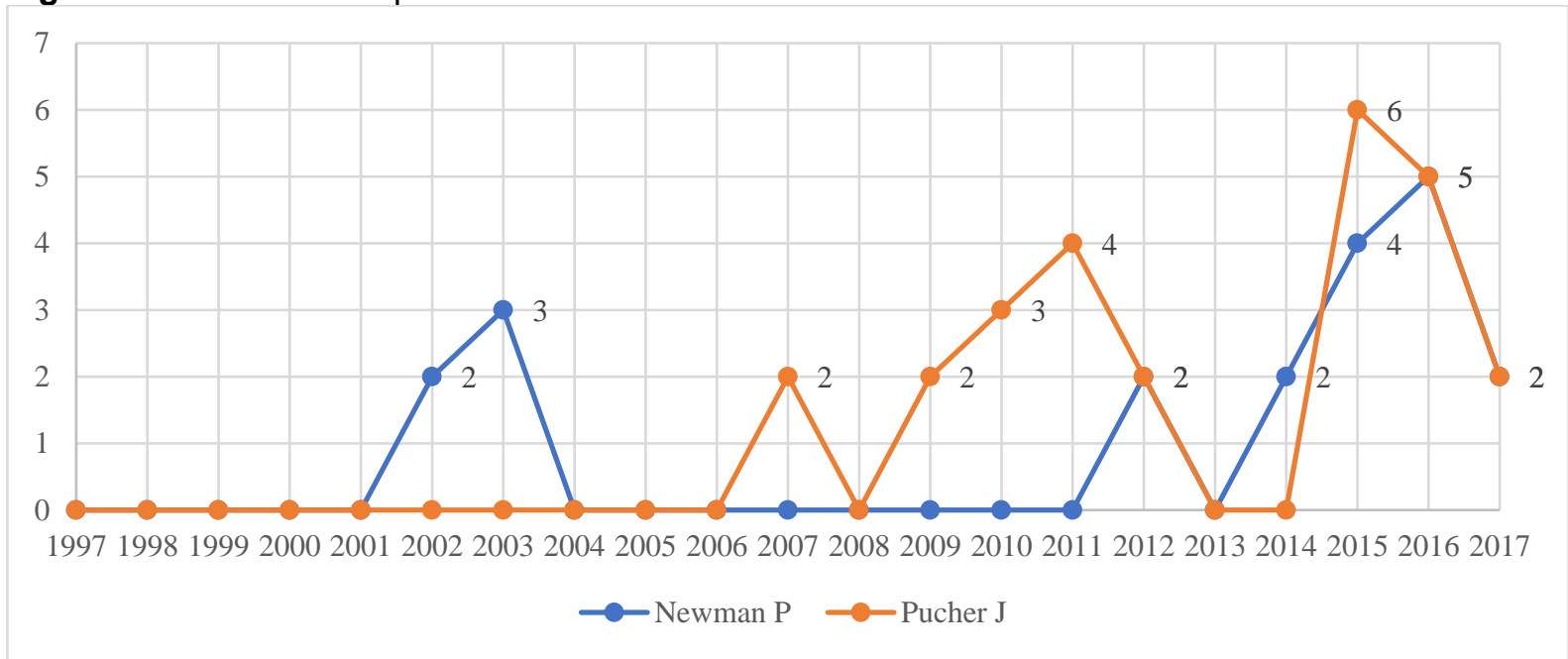

Fonte: Elaboração própria

\section{CONSIDERAÇÕES FINAIS}

Devido ao grande número de publicações que relacionam transporte público e sustentabilidade, torna-se imprescindível uma técnica quantitativa para analisar tais publicações. Por isso, é indicado uma análise bibliométrica para selecionar as publicações mais relevantes que abordem esses dois temas. No entanto, além da análise quantitativa, outra abordagem importante para se identificar tendências e padrões na literatura é a análise de rede social.

Assim, este artigo realizou uma análise bibliométrica das principais pesquisas sobre os temas, considerando três fatores: produtividade dos periódicos, produtividade dos autores e artigos mais citados. Com isso, selecionou-se 48 artigos que apresentaram diversas ramificações sobre os temas escolhidos.

Esses subtemas levantados e sumarizados na Tabela 4 apresentam convergência entre si, visto que podem ser relacionados de diversas formas, como: técnicas voltadas para melhoria do transporte público podem ser obtidas através de uma parceria público privada, ou ainda, a dependência do automóvel pode ser relacionada com políticas de restrição para uso deste e assim por diante.

Um dos pontos de destaque nessas publicações foi a caracterização do padrão de viagens relacionado a escolha modal. Ou seja, como o padrão de viagens e a escolha modal de um indivíduo ou um grupo de pessoas pode ser afetado por questões como: qualidade transporte público, idade, políticas de conscientização, etc. Outro ponto de destaque foram as publicações que levantaram técnicas para melhoria 
do transporte público, como uma melhor acessibilidade ou equidade do uso do solo, bem como pontos diretamente relacionados a sustentabilidade ambiental, social e financeira, mostrando assim a convergência da amostra selecionada com o tema.

Muitos artigos também tratam da dependência do automóvel como uma preocupação quando se trata de sustentabilidade, que acabam relacionando também com políticas para restrição do uso do automóvel. Em vista disso, a questão não é apenas disponibilizar o transporte público, mas também que ele seja com regularidade, que tenha capacidade de atender a população e que há uma necessidade de trabalhar com políticas de conscientização para que as pessoas priorizem o transporte não motorizado e o público.

Dessa forma, o transporte público por si só promove a sustentabilidade visto que permite a sua utilização em detrimento ao transporte individual, trazendo com isso vários outros aspectos positivos, como: melhor uso do solo, infraestrutura, mobilidade, mudanças no padrão de comportamento e de viagens, melhorias na produtividade do TP e assim, uma melhor viabilidade de implantação. Ou seja, a sua utilização não se reduz apenas a uma mera questão ambiental para a redução da emissão de $\mathrm{CO}_{2}$, mas também fatores relacionados a questões sociais e financeiras.

A análise de rede social permitiu identificar os principais países que apresentam as maiores publicações sobre esses temas: Estados Unidos, Inglaterra, Austrália e China. As palavras chaves com as maiores centralidades foram: desenvolvimento sustentável, ambiente construído, padrão de viagem, transporte público, mudanças climáticas, políticas, escolha modal, forma urbana e "a pé".

Tais palavras estão de acordo com o que foi sumarizado pela bibliometria, mostrando assim uma convergência entre essas duas análises. Como a centralidade é um indício de tendências da literatura, pode-se afirmar que uma possível tendência da literatura está relacionada com a sustentabilidade ambiental e social.

Por fim, conclui-se que a análise bibliométrica e a análise de rede social são complementares. A primeira mostra quais são as publicações mais relevantes em termos citações, periódicos e autores, a segunda apresenta uma abordagem que ajuda a identificar tendências de literatura, baseado em redes de co-citação. Assim, com base nas tendências das palavras chave e na sumarização do que foi encontrado pela bibliometria, os resultados foram convergentes. 


\section{REFERÊNCIAS}

ABOU-ZEID, M.; FUJII, S. Travel satisfaction effects of changes in public transport usage. Transportation, v. 43, n. 2, p. 301-314, 2016. https://doi.org/10.1007/s11116-015-9576-3

ADITJANDRA, P. T.; MULLEY, C. E.; NELSON, J. D. The influence of neighbourhood design on travel behaviour: Empirical evidence from North East England. Transport Policy, v. 26, p. 54-65, 2013. https://doi.org/10.1016/j.tranpol.2012.05.011

ALPKOKIN, P.; BLACK, J.; IYINAM, S.; E KESTEN, A. S. Historical analysis of economic, social and environmental impacts of the Europe-Asia crossings in Istanbul. International Journal of Sustainable Transportation, v. 10, n. 2, p. 65-75, 2016. https://doi.org/10.1080/15568318.2013.853852

ALPKOKIN, P., KIREMITCI, S. T., BLACK, J., E CETINAVCI, S. LRT and street tram policies and implementation in turkish cities, Journal of Transport Geography, v. 54, p.476-487, 2016. https://doi.org/10.1016/j.jtrangeo.2015.10.004

ARANTES, F. P., E SANTOS, T. F. Integração Da Cadeia De Suprimentos : Uma Análise Teórica E Bibliométrica das pulbicações. In: XXXVI ENCONTRO NACIONAL DE ENGENHARIA DE PRODUCÃO, 37., 2016, João Pessoa. Anais... João Pessoa-PB.

ARAÚJO, C. A. Bibliometria: evolução história e questões atuais. Em Questão, v. 12, n. 1, p. 11-32, 2006.

ARAÚJO, R. F., ALVARENGA, L.A. Bibliometria na pesquisa científica da pós-graduação brasileira de 1987 a 2007. Revista eletrônica de biblioteconomia e ciência da informação, v. 16, n. 31, 51-70. 2011

BLACK, J., PAEZ, A., E SUTHANAYA, P. A. Sustainable urban transportation: Performance indicators and some analytical approaches. Journal Of Urban Planning And

Development-Asce, v. 128, n. 4, 184-209, 2002. https://doi.org/10.1061/(ASCE)07339488(2002)128:4(184)

BLAINEY, S., HICKFORD, A., E PRESTON, J. Barriers to passenger rail use: a review of the evidence. Transport Reviews, v. 32, n. 6, p. 675-696, 2012.

https://doi.org/10.1080/01441647.2012.743489

BRIDA, J. G., DEIDDA, M., E PULINA, M. Tourism and transport systems in mountain environments: analysis of the economic efficiency of cableways in South Tyrol. Journal Of Transport Geography, v. 36, p. 1-11, 2014. https://doi.org/10.1016/i.jtrangeo.2014.02.004

BUEHLER, R. Promoting Public transportation comparison of passengers and policies in germany and the united states. Transportation Research Record, v. 2110, p. 60-68, 2009. https://doi.org/10.3141/2110-08

Determinants of transport mode choice: a comparison of Germany and the USA.

Journal of Transport Geography, v. 19, n. 4, p. 644-657, 2011.

https://doi.org/10.1016/i.jtrangeo.2010.07.005

BUEHLER, R., E PUCHER, J. Sustainable transport in freiburg: Lessons from germany's environmental capital. International Journal of Sustainable Transportation, v. 5, n.1, 4370, 2011a. https://doi.org/10.1080/15568311003650531 
Making public transport financially sustainable. Transport Policy, v. 18, n. 1, p. 126-138, 2011b. https://doi.org/10.1016/j.tranpol.2010.07.002

Cycling to work in 90 large American cities: New evidence on the role of bike paths and lanes. Transportation, v. 39, n. 2, p. 409-432. 2012. https://doi.org/10.1007/s11116-011$\underline{9355-8}$

BUEHLER, R.; PUCHER, J.; GERIKE, R.;GOTSCHI, T. Reducing car dependence in the heart of Europe: lessons from Germany, Austria, and Switzerland. Transport Reviews, v. 37, n. 1, p. 4-28, 2017. https://doi.org/10.1080/01441647.2016.1177799

CAMAGNI, R., GIBELLI, M. C., E RIGAMONTI, P. Urban mobility and urban form: the social and environmental costs of different patterns of urban expansion. Ecological Economics, v. 40, n. 2, 199-216, 2002. https://doi.org/10.1016/S0921-8009(01)00254-3

CANIELS, M. C. J., E ROMIJN, H. A. Strategic niche management: towards a policy tool for sustainable development. Technology Analysis \& Strategic Management, v. 20, n. 2, p. 245-266, 2008. https://doi.org/10.1080/09537320701711264

CARLSSON-KANYAMA, A., DREBORG, K. H., MOLL, H. C. C., E PADOVAN, D. D. Participative backcasting: a tool for involving stakeholders in local sustainability planning. Futures, v. 40, n. 1, 34-46, 2008. https://doi.org/10.1016/i.futures.2007.06.001

CHEN, C. The citespace manual. college of computing and informatics. Disponível em: http://cluster.uschool.drexel.edu/ cchen/citespace/CiteSpaceManual.pdf. 2014 Acesso em: 04 Maio 2017.

CHEN, C., SANJUAN, F. I., E HOU, J. The structure and dynamics of co T citation clusters: a multiple T perspective co T citation analysis. Journal of the American Society for Information Science and Technology, v. 7, p. 1386-1409, 2010. https://doi.org/10.1002/asi.21309

CHEN, H.; JIA, B.; LAU, S. S. Y. Sustainable urban form for chinese compact cities: challenges of a rapid urbanized economy. Habitat International, v. 32, n. 1, p. 28-40, 2008. https://doi.org/10.1016/j.habitatint.2007.06.005

CHERRY, C., CERVERO, R. Use characteristics and mode choice behavior of electric bike users in China. Transport Policy, v. 14, n. 3, p. 247-257, 2007.

https://doi.org/10.1016/i.tranpol.2007.02.005

CHESTER, M., PINCETL, S. ., ELIZABETH, Z. ., EISENSTEIN, W. ., E MATUTE, J. Infrastructure and automobile shifts: positioning transit to reduce life-cycle environmental impacts for urban sustainability goals. Environmental Research Letters, v. 8, n. 1, 2013. https://doi.org/10.1088/1748-9326/8/1/015041

COOPER, J., DONEGAN, K., RYLEY, T. J., SMYTH, A., E GRANZOW, E. Densification and urban compaction: reinforcing the drive for sustainability. Transportation Research Record, v. 1817 , p. 102-109, 2002. https://doi.org/10.3141/1817-13

CURRIE, G., E DELBOSC, A. Exploring public transport usage trends in an ageing population. Transportation, v. 37, n. 1, p. 151-164, 2010. https://doi.org/10.1007/s11116-009$\underline{9224-x}$

CURTIS, C. Planning for sustainable accessibility: The implementation challenge. Transport Policy, v. 15, v. 2, 2008. 
DAVISON, L.; ENOCH, M.; RYLEY, T. J.; QUDDUS, M.; WANG, C. A survey of demand responsive transport in Great Britain. Transport Policy, v. 31, p. 47-54, 2014.

https://doi.org/10.1016/j.tranpol.2013.11.004

DE GRUYTER, C., CURRIE, G., E ROSE, G. Sustainability measures of urban public transport in cities: a world review and focus on the Asia/Middle east region. Sustainability, v. 9, n. 1, 2017. https://doi.org/10.3390/su9010043

EGILMEZ, G.; TATARI, O. A dynamic modeling approach to highway sustainability: Strategies to reduce overall impact. Transportation Research Part A: Policy and Practice, v. 46, n. 7, p. 1086-1096, 2012. https://doi.org/10.1016/j.tra.2012.04.011

FINN, B.; NELSON, J. D. International perspective on the changing structure of the urban bus market. Transportation Research Record, v.1799, p.89-96, 2002.

https://doi.org/10.3141/1799-12

GILES-CORTI, B., et al. City planning and population health: a global challenge. Lancet, v. 388, n. 10062, p. 2912-2924, 2016. https://doi.org/10.1016/S0140-6736(16)30066-6

HAAPIO, A. Towards sustainable urban communities. Environmental Impact Assessment Review, v. 32, n. 1, 165-169, 2012. https://doi.org/10.1016/i.eiar.2011.08.002

HENSHER, D. A. Climate change, enhanced greenhouse gas emissions and passenger transport - What can we do to make a difference? Transportation Research Part D:

Transport and Environment, v. 13, n. 2, p. 95-111. 2008.

https://doi.org/10.1016/i.trd.2007.12.003

HICKMAN, R.; HALL, P.; BANISTER, D. Planning more for sustainable mobility. Journal of Transport Geography, v. 33, p. 210-219, 2013. https://doi.org/10.1016/.j.trangeo.2013.07.004

HOLDEN, E.; LINNERUD, K.; BANISTER, D. Sustainable passenger transport: Back to Brundtland. Transportation Research Part A: Policy and Practice, v. 54, p. 67-77., 2013. https://doi.org/10.1016/i.tra.2013.07.012

ISON, S.; MERKERT, R.; MULLEY, C. Policy approaches to public transport at airportsSome diverging evidence from the $\{U K\}$ and Australia. Transport Policy, v. 35, p. 265-274, 2014. https://doi.org/10.1016/j.tranpol.2014.06.005

JOSS, S.; MOLELLA, A. P. The eco-city as urban technology: perspectives on caofeidian international Eco-City (China). Journal of Urban Technology, v. 20, n. 1, p. 115-137, 2013. https://doi.org/10.1080/10630732.2012.735411

KAMRUZZAMAN, M. et al. Patterns of social capital associated with transit oriented development. Journal of Transport Geography, v. 35, p. 144-155, 2014. https://doi.org/10.1016/j.jtrangeo.2014.02.003

KENWORTHY, J. R. The eco-city: ten key transport and planning dimensions for sustainable city development. Environment and Urbanization, v. 18, n. 1, p. 67-85, 2008. https://doi.org/10.1177/0956247806063947

KIM, J.; FUJII, S.; LEE, B. Strategies to Promote Sustainable Mobility Management Incorporating Heterogeneity. International Journal of Sustainable Transportation, v. 7, n. 2, p. 107-124, 2013. https://doi.org/10.1080/15568318.2011.621099 
KOK, N.; JENNEN, M. The impact of energy labels and accessibility on office rents. Energy Policy, v. 46, p. 489-497, 2012. https://doi.org/10.1016/j.enpol.2012.04.015

MAY, A.; SHEPHERD, S.; TIMMS, P. M. Optimal transport strategies for European cities. Transportation, v. 27, n. 3, 285-315, 2000. https://doi.org/10.1023/A:1005274015858

MAY, A.; TRANTER, P. J.; E WARN, J. R. Progressing road safety through deep change and transformational leadership. Journal of Transport Geography, v. 19, n. 6, p. 1423-1430, 2011. https://doi.org/10.1016/j.jtrangeo.2011.07.002

MEINHOLD, J. L. et al. Social equity and environmental health: a search for solutions in portland, Oregon. International Journal of Social Sustainability in Economic, Social and Cultural Context, v. 8, n. 2, p. 144-159, 2013.

MILLER, D.; MERRILEES, B.;COGHLAN, A. Sustainable urban tourism: understanding and developing visitor pro-environmental behaviours. Journal of Sustainable Tourism, v. 23, n.1, p. 26-46, 2015. https://doi.org/10.1080/09669582.2014.912219

MILLER, P. et al. Public transportation and sustainability: a review. Ksce Journal of Civil Engineering, v. 20, n. 3, p. 1076-1083, 2016.

MURPHY, E., USHER, J. The Role of bicycle-sharing in the city: analysis of the irish experience. International Journal of Sustainable Transportation, v. 9, n. 2, p.116-125, 2015. https://doi.org/10.1080/15568318.2012.748855

MURRAY, A. T., DAVIS, R., STIMSON, R. J., E FERREIRA, L. Public transportation access. Transportation Research Part D: Transport and Environment, v. 3, n. 5, 319-328, 1998. https://doi.org/10.1016/S1361-9209(98)00010-8

NAIR, R. et al. Large-Scale Vehicle Sharing Systems: Analysis of Vélib'. International Journal of Sustainable Transportation, v. 7, n. 1, 85-106, 2013.

https://doi.org/10.1080/15568318.2012.660115

NAKANISHI, H.; BLACK, J. Social sustainability issues and older adults' dependence on automobiles in low-density environments. Sustainability (Switzerland), v. 7, n. 6, p.72897309, 2015. https://doi.org/10.3390/su7067289

Travel habit creation of the elderly and the transition to sustainable transport:

Exploratory research based on a retrospective survey. International Journal Of

Sustainable Transportation, v. 10, n. 7, 604-616, 2016.

https://doi.org/10.1080/15568318.2015.1059526

NEDER, R.; MARQUES, C. Aprendizagem Organizacional: Redes de Produção Científica sob a Perspectiva da Análise de Redes Sociais. In: 12th INTERNATIONAL CONFERENCE ON INFORMATION SYSTEMS \& TECHNOLOGY MANAGEMENT, 2015.

PRESTON, J. What's so funny about peace, love and transport integration? Research in Transportation Economics, v. 29, n. 1, p. 329-338, 2010.

https://doi.org/10.1016/j.retrec.2010.07.042

RICHARDSON, B. C. Sustainable transport: Analysis frameworks. Journal of Transport

Geography, v. 13, n. 1, p. 29-39, 2005. https://doi.org/10.1016/j.jtrangeo.2004.11.005 
RYLEY, T. J. et al. Sustainable development and airport surface access: the role of technological innovation and behavioral change. Sustainability (Switzerland), v. 5, n. 4, p. 1617-1631, 2013. https://doi.org/10.3390/su5041617

RYLEY, T. J. et al. Investigating the contribution of demand responsive transport to a sustainable local public transport system. Research in Transportation Economics, v. 48, p.364-372, 2014. https://doi.org/10.1016/j.retrec.2014.09.064

SALONEN, M.; TOIVONEN, T. Modelling travel time in urban networks: comparable measures for private car and public transport. Journal of Transport Geography, v. 31, p. 143-153, 2013. https://doi.org/10.1016/j.jtrangeo.2013.06.011

SANTOS, G., BEHRENDT, H., TEYTELBOYM, A. Part II: policy instruments for sustainable road transport. Research in Transportation Economics, v. 28, n. 1, p. 46-91, 2010.

SCOTT, J. Social Network Analysis. United Kingdom: Sage Publication, 2013.

SHAHEEN, S. A.; GUZMAN, S.; ZHANG, H. Bikesharing in Europe, the Americas, and Asia past, present, and future. Transportation Research Record, v. 2143, p.159-167, 2010. https://doi.org/10.3141/2143-20

SHIFTAN, Y.; KAPLAN, S.; HAKKERT, S. Scenario building as a tool for planning a sustainable transportation system. Transportation Research Part D: Transport and Environment, v.8, n. 5, p. 323-342, 2003. https://doi.org/10.1016/S1361-9209(03)00020-8

SONG, Y., HICKFORD, A., E PRESTON, J. Delivering sustainable public transport: the case of the better bus area fund. Research in Transportation Economics, v. 48, p.373-380, 2014. https://doi.org/10.1016/i.retrec.2014.09.066

TANIGUCHI, A., E FUJII, S. Promoting public transport using marketing techniques in mobility management and verifying their quantitative effects. Transportation, v. 34, p.1, 3749, 2007.

VIEGAS, J. M. Making urban road pricing acceptable and effective: searching for quality and equity in urban mobility. Transport Policy, v. 8, n. 4, p. 289-294, 2001.

https://doi.org/10.1016/S0967-070X(01)00024-5

WRIGHT, S., EMELE, C. D., FUKUMOTO, M., VELAGA, N. R., E NELSON, J. D. The design, management and operation of flexible transport systems: comparison of experience between UK, Japan and India. Research in Transportation Economics, v. 48, p. 330-338, 2014. https://doi.org/10.1016/j.retrec.2014.09.060

ZHOU, J. Sustainable commute in a car-dominant city: factors affecting alternative mode choices among university students. Transportation Research Part A: Policy and Practice, v. 46, n. 7, 1013-1029. https://doi.org/10.1016/j.tra.2012.04.001

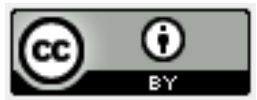

Artigo recebido em: 17/11/2017 e aceito para publicação em: 12/02/2019 DOI: http://dx.doi.org/10.14488/1676-1901.v19i1.3068 
Revista Produção Online. Florianópolis, SC, v. 19, n. 1, p. 44-71, 2019. 